Ann. Biol. anim. Bioch. Biophys., I970, 10 (I), I59-163.

\title{
RECHERCHES SUR LA DYNAMIQUE DES MODIFICATIONS QUANTITATIVES DE L'HÉMOGLOBINE ET DES ÉLÉMENTS FIGURÉS DU SANG CHEZ LES VEAUX ET LES JEUNES BOVINS
}

\author{
L. REBREANU \\ Faculté de Médecine vétérinaire, Timisoara, (Roumanie)
}

RÉSUMÉ

L'étude de la composition du sang chez 27 jeunes bovins de la race Bigarrée Roumaine, de la naissance à 12 mois, a permis de montrer que :

I. chez le Veau nouveau-né la quantité d'hémoglobine et lo nombre d'hématies et de leuco. cytes sont plus élevés que les mois suivants ;

2. le nombre de leucocytes diminue de deux mille environ le premier mois, puis continue à diminuer, mais plus lentement jusqu'à 12 mois ;

3. le nombre d'hématies diminue de 2 millions environ le premier mois, tend à augmenter jusqu'à cinq mois, puis diminue progressivement jusqu'à 12 mois.

L'hémoglobine subit une évolution semblable.

Ces résultats sont expliqués par le changement du mode d'oxygénation de l'organisme à la naissance et par les variations de la vitesse de croissance au cours des 12 premiers mois.

\section{INTRODUCTION}

L,e système biologique complexe que constitue le sang joue un rôle important dans de nombreux mécanismes métaboliques et l'évolution de sa composition peut refléter la dynamique des processus qui se développent dans 1'organisme du jeune.

Il est donc d'une grande importance théorique et pratique de connaître les modifications de la composition du sang : le nombre des hématies et la quantité d'hémoglobine permettent d'apprécier l'intensité des processus d'oxydation liés à la croissance et le nombre de leucocytes donne des indications sur les possibilités de défense de l'organisme. 
Les données sur la composition du sang des veaux sont peu précises.

WIRTH (I950) indique 7 à I4 millions d'hématies, I 2 à I5 mille leucocytes, I2, I à I4,6 $\mathrm{g}$ p. roo d'hémoglobine.

Pour Adamesteanu (I959) la quantité d'hémoglobine varie entre ir,9 et I3,6 $\mathrm{g}$ p. roo.

Pour Marek et Mocsy (I956) la quantité d'hémoglobine moyenne est de II g p. IOO.

Manta et al. (I96I) donnent les valeurs moyennes suivantes : hématies : 8,2 millions; leucocytes : 6,7 mille ; hémoglobine : II,37 g p. Ioo.

Mais aucun de ces auteurs ne donne de valeurs en fonction de l'âge. Or, on sait que la composition du sang varie avec l'âge. C'est la raison pour laquelle ces résultats ne concordent pas toujours entre eux.

Selon certaines données, les hématies et 1'hémoglobine diminueraient avec l'âge (MARUSKin, I960 ; Vintil Zaharova et LEBEDEV, I962 ; FEDOROV, I958 ; Filatovici, I959).

Pour d'autres auteurs (Novik, I957) le nombre d'hématies et la quantité d'hémoglobine augmenteraient progressivement de la naissance à II mois.

Enfin, pour KORJUEV (I957) la composition du sang subirait des fluctuations sans relation avec l'âge.

Nous avons donc entrepris une étude systématique de l'évolution de la composition du sang en fonction de l'âge des animaux.

\section{MATÉRIEL ETT MÉTHODES}

Les recherches ont été effectuées sur un lot de 27 bovins (I 6 mâles et I I femelles) de la race Bigarrée Roumaine, de la naissance à l'âge de I 2 mois.

Les prises de sang ont lieu chaque mois. Elles sont faites le matin avant la distribution de nourriture.

Les paramètres étudiés sont :

- nombre d'hématies déterminé avec la pipette de Potain;

- nombre de leucocytes déterminé avec la lame Goreaev;

- quantité d'hémoglobine à l'hémoglobinomètre de Sahli.

On a fait l'étude statistique des résultats d'après SNEDECOR (1957).

\section{RÉSULTATS}

Les valeurs observées sont présentées dans le tableau $\mathbf{I}$.

C'est à la naissance que le nombre d'hématies et de leucocytes et la quantité d'hémoglobine sont les plus élevés.

Le nombre d'hématies diminue d'environ deux millions le premier mois. Ensuite, il tend à augmenter jusqu'au cinquième mois, pour diminuer ensuite progressivement jusqu'à 12 mois.

L'hémoglobine subit une évolution semblable. 
TABLEAU I

Quantité d'hémoglobine, nombre d'hématies et de leucocytes des veaux et des jeunes bovins en fonction de l'âge

\begin{tabular}{|c|c|c|c|}
\hline Age (en mois) & $\begin{array}{l}\text { Hématies } \\
\text { (millions/ml) } \\
\bar{x} \pm \mathrm{S} \bar{x}\end{array}$ & $\begin{array}{l}\text { Leucocytes } \\
(\mathrm{mille} / \mathrm{ml}) \\
\bar{x} \pm \mathrm{S} \bar{x}\end{array}$ & $\begin{array}{c}\text { Hémoglobine } \\
\text { (g/100 ml de sang) } \\
\bar{x} \pm \mathrm{S} \vec{x}\end{array}$ \\
\hline 0 & $9,1 \pm 0,62$ & $12,3 \pm 0,21$ & $15,3 \pm 0,41$ \\
\hline 1 & $6,7 \pm 0,34$ & $10,4 \pm 0,27$ & $11,7 \pm 0,32$ \\
\hline 2 & $7,0 \pm 0,23$ & $9,1 \neq 0,18$ & $12,2 \pm 0,18$ \\
\hline 3 & $7,2 \pm 0,31$ & $9,6 \pm 0,22$ & $12,8 \pm 0,36$ \\
\hline 4 & $7,4 \pm 0,27$ & $8,8 \pm 0,31$ & $12,3 \pm 0,24$ \\
\hline 5 & $7,8 \pm 0,33$ & $9,9=0,25$ & $12,6 \pm 0,23$ \\
\hline 6 & $7,5 \pm 0,26$ & $9,6 \pm 0,09$ & $11,4 \pm 0,17$ \\
\hline 7 & $7,3 \pm 0,32$ & $9,9 \pm 0,16$ & $10,9 \pm 0,11$ \\
\hline 8 & $7,1 \pm 0,19$ & $9,1 \pm 0,12$ & $10,5 \pm 0,19$ \\
\hline 9 & $7,4 \pm 0,08$ & $8,9 \pm 0,18$ & $10,2 \pm 0,22$ \\
\hline 10 & $6,9 \pm 0,11$ & $8,7 \pm 0,23$ & $9,9 \pm 0,15$ \\
\hline 11 & $6,7 \pm 0,13$ & $8,4 \pm 0,15$ & $9,5 \pm 0,23$ \\
\hline 12 & $6,5 \pm 0,17$ & $8,1 \pm 0,21$ & $9,2 \pm 0,12$ \\
\hline
\end{tabular}

Le nombre de leucocytes diminue de 2 ooo environ le premier mois et continue à diminuer, mais moins rapidement au cours des mois suivants.

\section{DISCUSSION}

On peut penser que la richesse en hématies et en hémoglobine à la naissance est liée aux particularités du mode de ravitaillement en oxygène du fœetus pendant le développement intra-utérin. L'apport de quantités suffisantes d'oxygène se faisant grâce à l'abondance des hématies circulantes.

A la naissance, quand le mode d'oxygénation change brusquement, l'organisme passant d'un régime anoxémique à un régime riche en oxygène, l'augmentation du nombre des hématies s'arrête. La " faim » d'oxygène jouerait un rôle dans le déterminisme de l'hématopoïèse.

Après la naissance, les mécanismes apparus pendant la vie intra-utérine deviennent inutiles, ce qui détermine la diminution du nombre d'hématies et de la quantité d'hémoglobine. D'autre part l'hémoglobine fœtale est remplacée par une hémoglobine adulte.

L'augmentation du nombre d'hématies et de la quantité d'hémoglobine entre 2 et 5 mois coïncide avec une période de croissance importante.

La diminution du nombre des hématies et de la quantité d'hémoglobine entre 6 et I 2 mois peut être mise en relation avec une diminution de la vitesse de croissance et donc de l'intensité des processus métaboliques par rapport à la période précédente.

DAGAEVA et DAGAEV (I959) ont mis en évidence une corrélation positive entre la quantité d'hémoglobine et l'augmentation de poids des animaux. De même, EIDRI- 
CHEVICI (I958) trouve une relation positive entre l'intensité de croissance et les indices sanguins.

Pour Rubax (I96I) le nombre d'hématies et le pourcentage d'hémoglobine est un indice des processus de respiration cellulaire en relation étroite avec les processus oxydo-réducteurs.

La chute importante du nombre des leucocytes dans les premiers mois pourrait expliquer la faible résistance des veaux aux maladies pendant cette période.

\section{Reçu pour publication en septembre 1969.}

\section{SUMMARY}

\section{QUANTITATIVE CHANGES IN HAMOGLOBIN AND BLOOD CORPUSCLES IN YOUNG STEERS}

The blood composition of 27 steers from the Rumanian moltled breed was studied from birth to twelve months of age.

I. The rate of hæmoglobin and the numbers of hæmatids and leucocytes were higher at birth than later on.

2. The number of leucocytes decreased by 2000 during the first month and decreased on deceleratingly up the 12 months.

3. The number of hematids decreased by 2000 ooo during the first month, then tended to increase up to five months and progressively decreased up to 2 months.

The hemoglobin rate indergoes parallel alterations. The change in the mode of oxygenation at birth and the variations of growth rate during the first twelve months can account for these results.

\section{RÉFÉRENCES BIBLIOGRAPHIQUES}

Adamesteanu I., Nicolau A., Birza H., r959. Sémiologie médicale vétérinaire. Édit. Acad. R. P. R,

Dagaeva T. A., DAgaev M. M., 1959. Sur la connaissance du rapport entre les indices d'extérieur à ceux d'intérieur chez les bovins (en russe). Ref. J. biologhiia, 5, 27 I.

Eidrighevici E. V., r959. Importance des méthodes physiologiques dans l'appréciation de la productivité des animaux ayant comme but l'amélioration des races (en russe). Ref. J. biologhiia, 21, 27 I.

Filatovici V. V., 1959. Les modifications du nombre des éritrocytes et de la quantité d'hémoglobine dans le sang des veaux de la race Taghil (en russe). An. S. S. S. R. Uralksi filial T. In-ta biologhiia, 4, $7 \mathrm{r}-73$

Fedorov V. I., r958. La rythmicité de la croissance et son importance pratique (en russe). Jivotno vodstovo, $20(3), 49-5 \mathbf{I}$.

Korjuev P. A., i957. Le sang des animaux domestiques comme l'un des caractères d'ex térieur (en russe) Jurnal obscei biologhii, 2, I21-1 36 .

Limnitkaia I. I., I950. Les modifications des propriétés oxydatives du sang pendant la période embryonnaire (en russe). Izd. Akad. Nauk S. S. S. R, Moskva.

Maruskin A. P., I960. Les oscillations de la composition morphologique du sang chez les bovins en rapport à leur âge (en russe). Trudî Instituta movfologhii jivotnîh im. Severtova, 31, 85-87.

Manta D., Marian P., Palfy Fir., Bologh Gh., Nichita S., ig6r. Analyse d'une constance du sang chez le bétail de race Hereford importé chez nous. Travaux Scientifiques du I. A. P. G. C., Ed. Agro-Silvic., 259-263.

MAREK J., MocsY J., 1956. Lehrubuch der klinischen Diagnostik der inneren Krankheiten der Haustere Fischer, Jena.

Novik A. I., I957. Les indices sanguins chez la race Tachetée de noire de Bielorussie (en russe) Ref. J. Biologhiia, 24, 366.

Radughina Z. F., I965. Quelques indices sanguins chez les veaux dans le processus de la croissance (en russe). Veterinaria, 3, 69-7o. 
Ruban I. D., 196r. Types constitutionnels et tableau hématologique des bovins. Études et recherches de Biologie animale, 1, 7-1x.

SNedecor G. W., 1957. Statistical methods. Iowa State, College Press.

TARUK G. F., I96I. La dynamique de la quantité d'hémoglobine du sang des veaux de la race Siementhal en rapport à leur âge et conditions d'entretien (en russe). Ref. J. biologhiia, 12, 285:

Vintila I, CindeA A., I96I. Contributions à la connaissance d'une constance sanguine chez le jeune bétail et une de leurs corrélations à la production du lait de vache. Travaux scientifiques du $1 . A . T$., VI. 237-238.

WIRTH D., 1950. Grundlagen einer klnnischen Hamatologie der Hauäiere, Wien.

Zaharova F. V., Lebedev I. A., I962. L'influence du type et du niveau d'alimentation sur la composition du sang chez les bovins (en russe). Jivotnovodstov, $2,82-83$. 